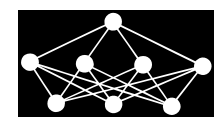

\title{
TOTAL ACCESSIBILITY NUMBER OF GRAPHS
}

\author{
E. Kılıç, P. Dündar*
}

\begin{abstract}
One of the most important problems in communication network design is the stability of network after any disruption of stations or links. Since a network can be modeled by a graph, this concept is examined under the view of vulnerability of graphs. There are many vulnerability measures that were defined in this sense. In recent years, measures have been defined over some vertices or edges having specific properties. These measures can be considered to be a second type of measures. Here we define a new measure of the second type called the total accessibility. This measure is based on accessible sets of a graph. In our study we give the total accessibility number of well known graph models such as $P_{n}, C_{n}, K_{m, n}, W_{1, n}$, $K_{1, n}$. We also examine this new measure under operations on graphs. A simple algorithm, which calculates the total accessibility number of graphs, is given. We observe that when any two graphs of the same size are compared in stability, it is inferred that the graph of higher total accessibility number is more stable than the other one. All the graphs considered in this paper are undirected, loopless and connected.
\end{abstract}

Key words: graph theory, accessibility number, total redundance, total accessibility

Received: February 28, 2014

DOI: $10.14311 / \mathrm{NNW} .2017 .27 .017$ Revised and accepted: July 10, 2017

\section{Introduction}

A network can be modeled by a graph whose vertices represent the stations, the processors etc. and whose edges represent the lines of communication links, roads, paths etc. The vulnerability measures the resistance of the network to any disruption in its stations or in its communication links. It is also another subject area to examine the remained network for failures after any disruption. Thus, the construction of a communication network plays an important role in the efficiency of the network. Communication networks can be modeled by connected graphs. The vulnerability of a graph is the resistance of the graph keeping some of its properties after any disruptions in any number of vertices or edges [1]. In the graph theory many well known vulnerability measures such as connectivity, edge connectivity, toughness, integrity, tenacity, and neighbor-integrity were studied widely in the past to describe the stability of communication networks [2-6]. Since any network

\footnotetext{
*Elgin Kılıç - Corresponding author; Pınar Dündar; Ege University, Faculty of Science, Department of Mathematics, Turkey, E-mail: elgin.kilic@ege.edu.tr, pinar.dundar@ege.edu.tr
} 
can be modeled by a graph, the vulnerability of a network can be examined by some measures defined in graphs $[11,14,16]$. But all these measures work within the whole vertex set or edge set of the graph. Recently, many theorists of graphs have begun to work on some measures which work on vertices or edges of the graph that have a specific property, for example a sub set of vertices of the graph which is a dominating set $[12,13,15]$. In this paper, the total accessibility, which is a new measure as the second type of measures mentioned above, is defined. It is believed that such measures are more convenient. One of those measures is the total redundancy depending on dominating sets of a graph [10]. The accessibility number of graphs using the neighborhood concept is defined and some results have already been published in [7]. We define the total accessibility number of a graph by using the vertices in accessible sets of the graph.

A graph $G$ is denoted by $G=(V(G), E(G))$, where $V(G)$ and $E(G)$ represent the set of vertices and the set of edges of $G$, respectively.

Definition 1. A spanning graph is a subgraph of any graph $G$ which contains all the vertices of $G$.

Definition 2. In a graph $G=(V, E)$, the open neighborhood of vertex $v \in V$ is $N(v)=\{x \in V \mid v x \in E\}$, the set of vertices adjacent to $v$.

Definition 3. The closed neighborhood of a vertex $v \in V$ is denoted by $N[v]=N(v) \cup\{v\}$.

Definition 4. For a set $S$ of vertices, the neighborhood of $S$ is the union of the neighborhoods of the vertices, and so it is the set of all vertices adjacent to at least one member of $S$.

Definition 5. If $G=(V, E)$ is a connected graph, the distance $d(u, v)$ between two vertices $u$ and $v$ of $G$ is defined as the minimum length of a $u, v$ path of $G$.

Definition 6. The eccentricity $e(v)$ of $v$ is $\max _{u \in V} d(u, v)$. That is, $e(v)$ is the distance between $v$ and a vertex farthest from $v$.

Definition 7. The radius $\operatorname{rad} G$ of $G$ is the minimum eccentricity among the vertices of $G$.

Definition 8. A set $S \subset V$ is a dominating set if every vertex in $V$ is either in $S$ or is adjacent to a vertex in $S$, that is $V=\cup_{s \in S} N[s]$.

Definition 9. The domination number $\gamma(G)$ is the minimum cardinality of a dominating set.

Definition 10. Let $G=(V, E)$ be a connected graph for $\forall x \in V-S$, if vertex $x$ is adjacent to set $S, N(S)$ or $N[S]$, then set $S \subset V$ is an accessible set of graph $G$. Accessibility number is the minimum number cardinality of over all accessible sets of graph $G$ and denoted by $\eta(G)[8,9]$.

Here are some results on the accessibility number of some graph models: 
Result 1. For path $P_{n}, \eta\left(P_{n}\right)=\left\lfloor\frac{n}{5}\right\rfloor+1$.

Result 2. For cycle $C_{n}, \eta\left(C_{n}\right)=\left\lfloor\frac{n}{5}\right\rfloor+1$.

Result 3. For complete graph $K_{n}, \eta\left(K_{n}\right)=1$.

Result 4. For complete bipartite graph $K_{m, n}, \eta\left(K_{m, n}\right)=1$.

Result 5. For wheel graph $W_{1, n-1}, \eta\left(W_{1, n-1}\right)=1$.

Result 6. For star graph $K_{1, n-1}, \eta\left(K_{1, n-1}\right)=1$.

\section{Total accessibility number}

Definition 11. Let $G$ be a connected graph. The total accessibility number of $G$ is defined as

$$
\operatorname{TA}(G)=\min \left\{\sum_{v \in S}(2+\operatorname{deg}(v)): S \subseteq V \wedge|N[x] \cap N[S]| \leq 1 \forall x \in V\right\} .
$$

Theorem 12. If in any connected $\operatorname{graph} G \operatorname{rad} G \leq 4$ and $X$ be the set of central vertices, then

$$
\mathrm{TA}(G)=\sum_{v \in X} \operatorname{deg}(v) .
$$

Theorem 13. If any spanning subgraph of graph $G$ is a star graph, then

$$
\mathrm{TA}(G)=\delta(G)+2
$$

Proof. If a graph contains a star as a subgraph, every vertex of the graph is accessible (reachable) from central vertex. Hence the necessity in the TA definition is met. By the way, since the TA value is expected to be lower we need to chose the vertex that is of the minimum degree. This minimum degree corresponds to $\delta$ of the graph.

The results on total accessibility number of well known graph models are listed below:

Result 1. For $P_{n}$,

$$
\operatorname{TA}\left(P_{n}\right)= \begin{cases}4 \cdot \eta\left(P_{n}\right), & n \equiv 0,4(\bmod 5) \\ 4 \cdot \eta\left(P_{n}\right)-1, & n \equiv 2,3(\bmod 5) \\ 4 \cdot \eta\left(P_{n}\right)-2, & n \equiv 1(\bmod 5)\end{cases}
$$

Result 2. For $C_{n}, \mathrm{TA}\left(C_{n}\right)=4 \cdot \eta C_{n}$.

Result 3. For complete bipartite graph $K_{m, n}, \operatorname{TA}\left(K_{m, n}\right)=2+\min \{m, n\}$.

Result 4. For wheel graph $W_{1, n-1}, \mathrm{TA}\left(W_{1, n-1}\right)=5$.

Result 5. For star graph $K_{1, n-1}, \mathrm{TA}\left(K_{1, n-1}\right)=1$. 


\section{Graph operations and total accessibility number}

Let $G_{1}=\left(V_{1}, E_{1}\right)$ and $G_{2}=\left(V_{2}, E_{2}\right)$ be two graphs of having different orders, no common vertices and with no isolated vertex.

Definition 14. The union $G=G_{1} \cup G_{2}$ of graphs $G_{1}$ and $G_{2}$ with disjoint vertex sets $V_{1}$ and $V_{2}$ and edge sets $E_{1}$ and $E_{2}$ is the graph with $V_{1} \cup V_{2}$ and $E_{1} \cup E_{2}$.

Definition 15. The join $G=G_{1}+G_{2}$ of graphs $G_{1}$ and $G_{2}$ with disjoint point sets $V_{1}$ and $V_{2}$ and edge sets $E_{1}$ and $E_{2}$ is the graph union $G_{1} \cup G_{2}$ together with all the edges joining $V_{1}$ and $V_{2}$.

Definition 16. The cartesian product $G_{1} \cdot G_{2}$ of graphs $G_{1}$ and $G_{2}$ is a graph such that the vertex set of $G_{1} \cdot G_{2}$ is the cartesian product $V\left(G_{1}\right) \cdot V\left(G_{2}\right)$; and any two vertices $\left(u, u^{\prime}\right)$ and $\left(v, v^{\prime}\right)$ are adjacent in $G_{1} \cdot G_{2}$ if and only if either

$$
\begin{gathered}
u=v \text { and } u^{\prime} \text { is adjacent with } v^{\prime} \text { in } G_{2} \text {, or } \\
u^{\prime}=v^{\prime} \text { and } u \text { is adjacent with } v \text { in } G_{1} .
\end{gathered}
$$

Theorem 17. Total accessibility number of the union of graphs $G_{1}$ and $G_{2}$ is

$$
\operatorname{TA}\left(G_{1} \cup G_{2}\right)=\operatorname{TA}\left(G_{1}\right)+\operatorname{TA}\left(G_{2}\right) .
$$

Theorem 18. Total accessibility number of the union of graphs $G_{1}, G_{2}, \ldots$, $G_{m-1}, G_{m}$ is

$$
\mathrm{TA}\left(G_{1} \cup \cdots \cup G_{m}\right)=\operatorname{TA}\left(G_{1}\right)+\cdots+\mathrm{TA}\left(G_{m}\right) .
$$

Theorem 19. For $P_{n}$ and $P_{m}$, where $m<n$, $\mathrm{TA}\left(P_{n}+P_{m}\right)=m+3$.

Proof. Since $m<n$, the vertex of the optimal accessible set have to be chosen from $P_{n}$. In this case the number and degrees of these vertices will be lower. Let us choose vertex $v$ from $P_{n}$, which is connected to vertex $u$ in $P_{m}$. The vertex degree of $v$ is equal to $2+m$ or $1+m$ at least. In both cases the rest of all vertices will be connected to the neighborhood of vertex $v$. Thus $\mathrm{TA}\left(P_{m}+P_{n}\right)=(1+m)+2=$ $m+3$.

Theorem 20. For $K_{1, n-1}$ and $K_{1, m-1}$, where $n<m$,

$$
\operatorname{TA}\left(K_{1, n-1}+K_{1, m-1}\right) \geq \operatorname{TA}\left(K_{1, n-1}\right)+\operatorname{TA}\left(K_{1, m-1}\right) .
$$

Proof. Due to the definition of TA, we need to choose the vertices from the graph $K_{1, m-1}$ which will form the accessible set since degree of any vertex will be lower in this set. Thus $\operatorname{deg}(v)=1+m$. Since $n<m$, it is better to choose the vertex $v$ which satisfies $\operatorname{deg}(v)=1+n$. Then $\operatorname{TA}\left(K_{1, n-1}+K_{1, m-1}\right)=(1+m)+1=2+m$ is obtained. Also $\mathrm{TA}\left(K_{1, n-1}\right)=1+2$ and $\mathrm{TA}\left(K_{1, m-1}\right)=1+2$.

Theorem 21. For $W_{1, n-1}$ and $W_{1, m-1}$ where $n<m$, 


$$
\mathrm{TA}\left(W_{1, n-1}+W_{1, m-1}\right) \geq \mathrm{TA}\left(W_{1, n-1}\right)+\mathrm{TA}\left(W_{1, m-1}\right) .
$$

Proof. The vertex having a minimal degree is chosen from $W_{1, m-1}$ to construct the accessible set. In this case every chosen vertex $v$ satisfies $\operatorname{deg}(v)=n$. Hence $\mathrm{TA}\left(W_{1, n-1}+W_{1, m-1}\right)=(n+2)+3=n+6$ is obtained. Also $\mathrm{TA}\left(W_{1, n-1}\right)=3+2=$ 5 and $\operatorname{TA}\left(W_{1, m-1}\right)=3+2=5$. Then it is clear that $\operatorname{TA}\left(W_{1, n-1}+W_{1, m-1}\right) \geq$ $\mathrm{TA}\left(W_{1, n-1}\right)+\mathrm{TA}\left(W_{1, m-1}\right)$.

Theorem 22. For $K_{m, n}$ and $K_{p, r}$, where $m<n<p<r$,

$$
\mathrm{TA}\left(K_{m, n}+K_{p, r}\right) \geq \mathrm{TA}\left(K_{m, n}\right)+\mathrm{TA}\left(K_{p, r}\right) .
$$

Proof. Let us do the proof in an algebraic way. Our purpose is to find general values for left-hand side and right-hand side of the inequality. To obtain the optimal value of $\mathrm{TA}\left(K_{m, n}+K_{p, r}\right)$, each element of accessible set should be chosen from the graph $K_{p, r}$. Hence the degree of each chosen vertex is $(m+n)+p$ and TA value is $m+n+p+2$ in this case. If we take into consideration TA values of $K_{m, n}, K_{p, r}$ graphs separately, we obtain $m+2$ and $p+2$ respectively. Finally $\mathrm{TA}\left(K_{m, n}\right)+\mathrm{TA}\left(K_{p, r}\right)=m+p+4$ from the definition of bipartite graphs $n \geq 2$. Therefore we have $m+n+p+2 \geq m+p+4$.

Theorem 23. For $P_{m}$ and $P_{n}$, where $m=3$,

$$
\mathrm{TA}\left(P_{3} \cdot P_{n}\right)<4 \cdot \eta\left(P_{n}\right) .
$$

Proof. Since the resulting graph after the product operation has a lattice structure, we need to choose vertices from the vertex set lying in the middle of the graph to find the optimal value of TA. The set of vertices of this selection corresponds to the accessibility number of $P_{n}$. And the vertex degree of each vertex in this set is 4. From the definition of TA we can obtain $4 \cdot \eta\left(P_{n}\right)$ at most. The vertex degree of at most 2 vertices in this accessible set can be 3 . In this case we have an inequality form instead of an equality one. Thus $\operatorname{TA}\left(P_{3} \cdot P_{n}\right)<4 . \eta\left(P_{n}\right)$.

Theorem 24. For $C_{n}$ and $C_{m}$,

$$
\mathrm{TA}\left(C_{n} \cdot C_{m}\right)=\eta\left(C_{m} \cdot C_{n}\right) \cdot 6 .
$$

Proof. All graphs resulting from $C_{n} \cdot C_{m}$ produce 4-regular graphs. In any cycle each vertex is connected to 2 other vertices. When this is taken into consideration, after product operation each vertex has degree 4. If the accessible number of this new resulting graph is found, it is easy to calculate the TA value of this new graph since it is a 4-regular. From the definition of TA for each vertex we add 2 and then $\mathrm{TA}\left(C_{n} \cdot C_{m}\right)=\eta\left(C_{m} \cdot C_{n}\right) \cdot 6$.

\section{Algorithm for total accessibility number of a graph}

The pseudocode for computing the total accessibility number of a graph is given in Algorithm 1. It uses the adjacency matrix of graph $G$. With the help of adjacency 
matrix, a distance matrix of the graph is constructed. The accessible sets of the graphs are found by making a search on the distance matrix of the graph where the distance between any vertex pair is 2 at most. After finding the accessible sets of the graph, the sum of degrees of vertices in every accessible set is calculated. Then the accessible set, which has the minimum value, is taken.

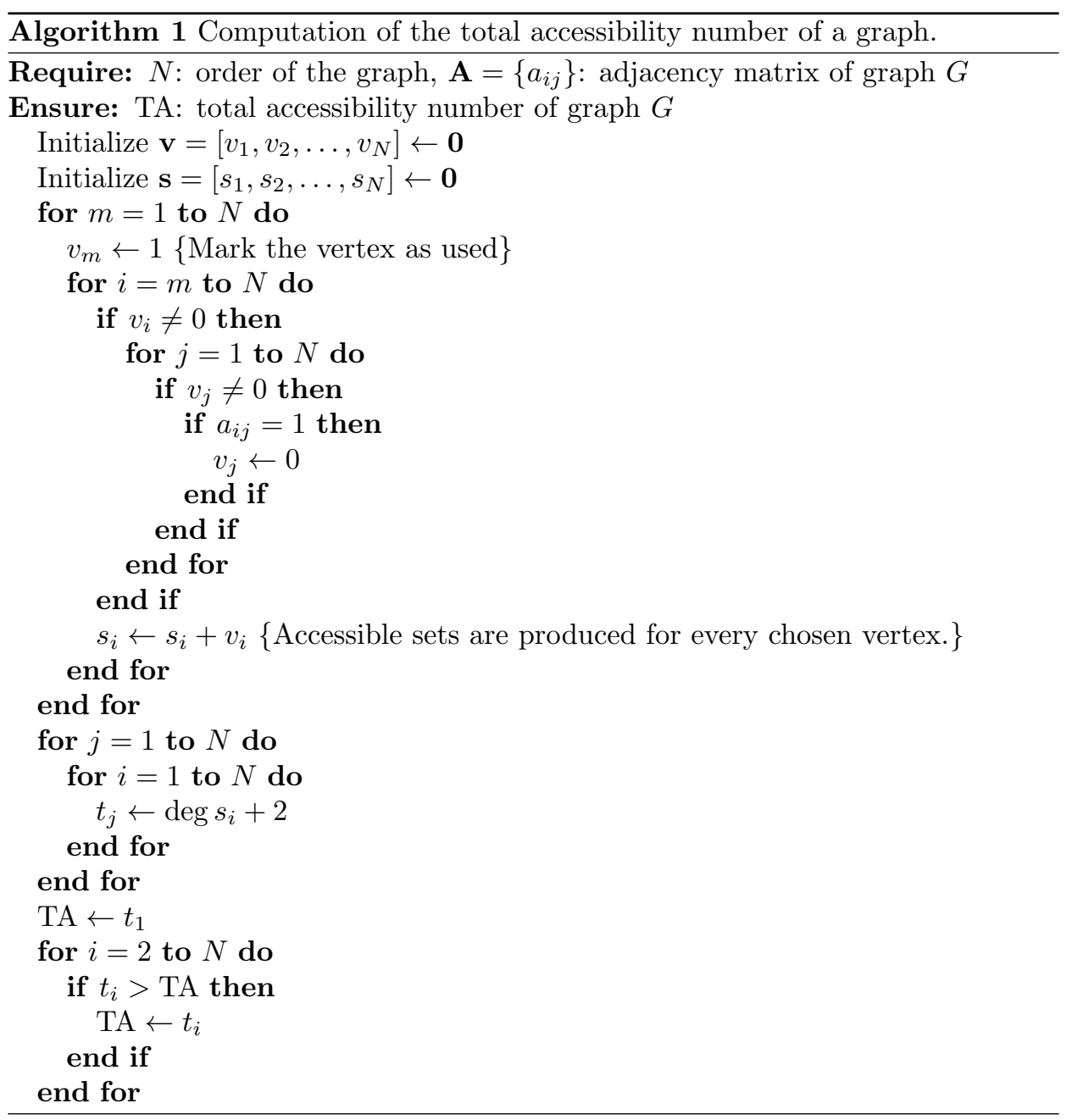

\section{Conclusion}

In this study, a new vulnerability measure called the total accessibility number (based on accessible sets) is defined. The total accessibility numbers of some graphs such as $P_{n}, C_{n}, K_{m, n}, W_{1, n-1}, K_{1, n-1}$ are examined. In communication networks after any disruption on stations, the stability of remaining components plays a great part in network design. In this sense, the connection in remaining components can 
be examined on accessible sets. When two graphs that have the same order are compared for stability, in some cases the graph, whose TA value is higher, is more stable and reliable then the other one. This means, the communication range in remaining components of the network is more dense after any breakdown in the network whose TA value is higher. We intend to study directed graphs and trees in order to give exact bounds for the total accessibility number. Our next target is to improve the algorithm for graphs of larger size.

\section{References}

[1] BAGGA K.S., BEINEKE L.W, LIPMAN M.J., PIPPERT R.E. Edge-integrity: A survey. Discrete Math. 1994, 124(1-3), pp. 3-12, doi: 10.1016/0012-365X (94)90084-1.

[2] BAGGA K.S., BEINEKE L.W., LIPMAN M.J., PIPPERT R.E., SEDLMEYER R.L. A good algorithm for the computation of the edge-integrity of trees. In: 19th Southeastern Conference on Combinatorics, Graph Theory, and Computing, Baton Rouge, LA. 1988, 67, pp. 225-232.

[3] BAREFOOT C.A., ENTRINGER R., SWART H. Vulnerability in graphs - A comparative survey. J. Combin. Math. Combin. Comput. 1987, 1(38), pp. 13-22.

[4] BEINEKE L.W., OELlERMANn O.R., PIPPERT R.E. The Average Connectivity of A Graph. Discrete Math. 2002, 252(1-3), pp. 31-45, doi: 10.1016/S0012-365X(01)00180-7.

[5] BUCKLEY F., HARARY F. Distance in Graphs. California: Addison Wesley Pub., 1990.

[6] DÜNDAR P., Aytac A. Integrity of Total Graphs via Certain Parameters. Mathematical Notes. 2004, 76(5-6), pp. 665-672, doi: 10.1023/B:MATN.0000049665.92885.26.

[7] DÜNDAR P. The stability measures of static interconnection networks and binary trees. In: Proceeding of the Third International Symposium on Computer Networks, Turkey. 1998, pp. 240-245.

[8] DÜNDAR P. Stability measures of some static interconnection networks. Int.J. Comput. Math. 2001, 76(4), pp. 455-462, doi: 10.1080/00207160108805039.

[9] DÜNDAR P. Accessibility Number and The Neighbour-integrity of Generalised Petersen Graphs. Neural Networks World. 2001, 11(2), pp. 167-174.

[10] GODDARD W., OELLERMANN O.R., SLATER P.J., SWART H.C. Bounds on the total redundance and efficiency of a graph. Ars. Combin. 2000, 54, pp. 129-138.

[11] HARARY F. Graph Theory. California: Addison Wesley Pub., 1969.

[12] HAYNES T.W., HENNING M.A., HOWARD J. Locating and total dominating sets in trees. Discrete Applied Math. 2006, 154(8), pp. 1293-1300, doi: 10.1016/j.dam.2006.01.002.

[13] HENNING M.A., OELlERMANn O.R., SWART H.C. Distance Domination critical graphs. J. Combin. Math. Combin. Comput. 2003, 44, pp. 33-45.

[14] LESNIAK L., CHARTRAND G. Graphs and Digraphs. 2nd ed. California: Wadsworth Publishing, 1986.

[15] van der MERWE L.C., MYNHARDT C.M., HAYNES T.W. Total domination edge critical graphs with maximum diameter. Discuss. Math. Graph theory. 2001, 21(2), pp. 187-205, doi: 10.7151/dmgt.1143.

[16] WEST D.B. Introduction to Graph Theory. NJ: Prentice Hall, 2001. 